\title{
Retrograde facilitation under midazolam: The role of general and specific interference
}

\author{
Lynne M. Reder, Joyce M. OAtes, Daniel Dickison, and John R. Anderson \\ Carnegie Mellon University, Pittsburgh, Pennsylvania \\ Ferenc Gyulai and Joseph J. Quinlan \\ University of Pittsburgh, Pittsburgh, Pennsylvania \\ JENNIFER L. FerRIS \\ Carnegie Mellon University, Pittsburgh, Pennsylvania \\ AND \\ Michael Dulik and Brandi F. JefFerson \\ University of Pittsburgh, Pittsburgh, Pennsylvania
}

\begin{abstract}
In a double-blind, placebo-controlled experiment that used midazolam, a benzodiazepine that creates temporary amnesia, we compared acquisition and retention of paired associates of different types. Some word pairs were studied before the injection of saline or midazolam, and two lists of word pairs were studied after the injection. Critical comparisons involved retention of pairs that were practiced on all three lists, pairs studied on only one list, and pairs that involved recombining cue and response terms from one list to the next, as a function of drug condition. Previous research with benzodiazepines had found retrograde facilitation for material acquired prior to injection, compared with the control condition. One explanation for this facilitation is that the anterograde amnesia produced by the benzodiazepine frees up the hippocampus to better consolidate previously learned material (Wixted, 2004, 2005). We accounted for a rich data set using a simple computational model that incorporated interference effects (cue overload) at retrieval for both general (experimental context) interference and specific (stimulus term) interference without the need to postulate a role for consolidation. The computational model as an Excel spreadsheet may be downloaded from www.psychonomic.org/archive.
\end{abstract}

Psychologists have long investigated the class of mechanisms that affect retention of past experience. Wixted $(2004,2005)$ notes that psychologists have ignored the role of consolidation while debating the role of interference and decay as mechanisms of forgetting. He reviews evidence from psychology, psychopharmacology, and neuroscience to argue that the traditional psychological theories of forgetting "may not be relevant to the kind of interference that induces most forgetting in everyday life" (p. 6). Wixted reviews evidence from psychopharmacology to support the claim that general interference or "mental exertion" is a major determinant in whether information is forgotten. In particular he notes that benzodiazepines, which produce amnesia for material learned after the drug, create retrograde facilitation for material learned before the drug. He argues that this results from the absence of mental exertion. This article reports a new study designed to understand the mechanisms that underlie the retrograde facilitation observed under the influence of benzodiazepines.

Studies using benzodiazepines, such as diazepam (Valium) and alprazolam (Xanax), as well as alcohol, have been used to induce temporary anterograde amnesia. Information presented after ingesting this drug tends not to be learned; however, information acquired prior to ingesting the drug is actually better retained than it would have been had the subjects received the control (saline) instead of the drug. This retrograde facilitation was interpreted as resulting from enhanced consolidation for material acquired prior to the amnesia. Wixted (2004) writes,

To summarize, sleep, alcohol, and benzodiazepines all result in retrograde enhancement of memory, and, theoretically, they all do so for the same reason: The reduced rate of memory formation protects recently formed memories from interference, interference that would otherwise arise because of the demands placed on a limited-resource hippocampal system (p. 257).

The studies that have examined the effect of benzodiazepines on memory have compared retention of lists studied prior to injection of drug versus saline, as well as - more obviously - the retention of lists given postinjection. However, those studies involved free recall and thus were not

L.M. Reder, reder@cmu.edu 
able to compare retention of preinjection items as a function of the type of items learned postinjection. If retrograde facilitation results from a reduced rate of memory formation postinjection, the type of information acquired should not necessarily matter. An alternative account that we propose posits that the facilitation for the items acquired preinjection results from a reduction in interference rather than an increase in the ability to consolidate.

The experiment reported here compares these conditions using the drug midazolam, a benzodiazepine that produces transient anterograde amnesia. It is a fast-acting anxiolytic used routinely in medical procedures, including dental and pediatric surgeries. In a cued-recall task, retention of a list studied prior to drug injection is compared with performance on a list studied prior to an injection of saline. The experiment uses a double-blind, within-subjects design (subjects get saline one day and midazolam another). Subjects study word pairs, and their cued-recall accuracy and latency for correct responses is measured. Of particular interest is performance on the item pairs from the list given prior to the injection (List 1) as a function of drug condition.

The pairs do not differ prior to injection. It is their postinjection treatment that differentiates them. Specifically, one third of the pairs - practice pairs - are repeated on each list, one third - control pairs - are only studied on one of the three lists, and the other third have a changed cue-to-response mapping from list to list. When pairs are learned with a saline injection, we expect final cued-recall performance to be best for the practiced pairs and worst for the "cue-overload" pairs - that is, the ones for which there are multiple responses for each cue. (Which one is to be recalled at test depends on the list cue provided along with the stimulus word.) The pairs seen in only one list are expected to be intermediate in performance. Of interest is how these various conditions are affected by midazolam and how retention of List 1 pairs learned prior to the injection differs as a function of drug condition and type of pair. We developed a simulation of the experiment to try to account for the cued-recall performance (including response times and errors at final test) for the different pair types in both drug conditions. Of particular interest was whether we needed to posit a role for consolidation to explain the empirical results.

\section{METHOD}

\footnotetext{
Subjects

Thirty-one healthy volunteers between 18 and 35 years old participated for a payment of $\$ 150$ each. All were screened by a doctor, and all gave their written informed consent for a protocol approved by the institutional review boards of Carnegie Mellon University and the University of Pittsburgh.

\section{Design, Materials, and Procedure}

Seventeen of the subjects received midazolam in their first session and 14 received saline. Assignment of drug condition to session was randomly determined and unknown to subject or staff at the hospital (only the pharmacy and the principal investigator knew the assignment). The slight imbalance in order effects was due to unforeseen attrition in subjects due to their falling asleep from the sedative. In
}

other respects the sessions were identical, except that different words were used for each session.

During the acquisition phase, subjects studied 45 word pairs on each of three separate lists. The first word of the pair served as a cue to recall the second word of the pair. Each list had 3 types of pairs, 15 of each: practice pairs repeated on all 3 lists, control pairs studied on only that list, and interference pairs with different response terms assigned to the cue word than used on the other lists (these response terms were used with other cue words on the other lists). Words were randomly assigned to pairs and condition for each subject.

Subjects sat upright on an inclined hospital bed with a laptop computer placed on a table positioned for easy viewing and responding. List 1 was studied and tested on the laptop before the injection was given, although the i.v. catheter was already in place. Each word pair was displayed on the screen for $3 \mathrm{sec}$. Following study there were two test-study cycles of the 45 word pairs. For each pair, a cue word was displayed and the subject tried to type in the response term associated with that cue (in the list just studied, in the case of interference pairs). Subjects could hit the Return key if unsure of the response. Regardless of the response, the word pair was re-presented for an additional $2.5 \mathrm{sec}$ of study. After all pairs were tested, the 45 pairs were tested again in a different random order.

Following the test-study cycles for List 1, subjects were hooked up to monitoring equipment and the injection was administered. A nurse monitored vital signs during acquisition of the two remaining lists. After the injection, subjects studied the pairs for List 2 with the same study, test-study procedure used for List 1 . The same procedure was repeated for List 3 after completing the List 2 test-study cycles. Each list was named at the beginning of study of that list, and subjects were informed that it was important to note the list for the final test. Each test-study phase lasted approximately $17 \mathrm{~min}$. After completing the test-study phase for List 3, subjects were disconnected from the monitoring equipment and escorted to the hospital cafeteria for a snack.

Approximately $1 \mathrm{~h}$ after the injection of midazolam or saline, subjects began the final test phase. In this phase, a test trial consisted of the first word of a studied pair, plus the name of the list on which the pair had appeared. Each pair was tested only once and no feedback was provided. Since practice pairs appeared on all 3 lists, one third was randomly selected to be tested for each of the 3 lists.

\section{RESULTS}

\section{Acquisition Performance}

Figure 1 presents the acquisition data for each list for each type of pair as a function of drug condition. ${ }^{1}$ For List 1 , there was neither an effect of drug condition nor an effect of type of word pair, because the subjects had not yet received an injection and word pairs would only differ on subsequent lists.

Lists 2 and 3 show clear effects of drug manipulation for the control and interference pairs $[F(1,30)=108.1$, $p<.001$ and $F(1,30)=72.4, p<.001$, respectively], such that pairs were not learned well after an injection of midazolam. There was also a significant drug $\times$ list interaction $[F(5,150)=13.4, p<.001]$, reflecting the fact that practice pairs were studied preinjection, and therefore were less affected by midazolam. List 3 shows the same pattern as that for List 2, but the effects were not quite as strong, suggesting that the effects of midazolam began to wear off by List 3 . There was also a significant drug $\times$ list $\times$ pair interaction $[F(1,30)=6.3, p<.05]$, such that the deleterious effects of midazolam were reduced for acquisition of List 3 compared with List 2 for the control 


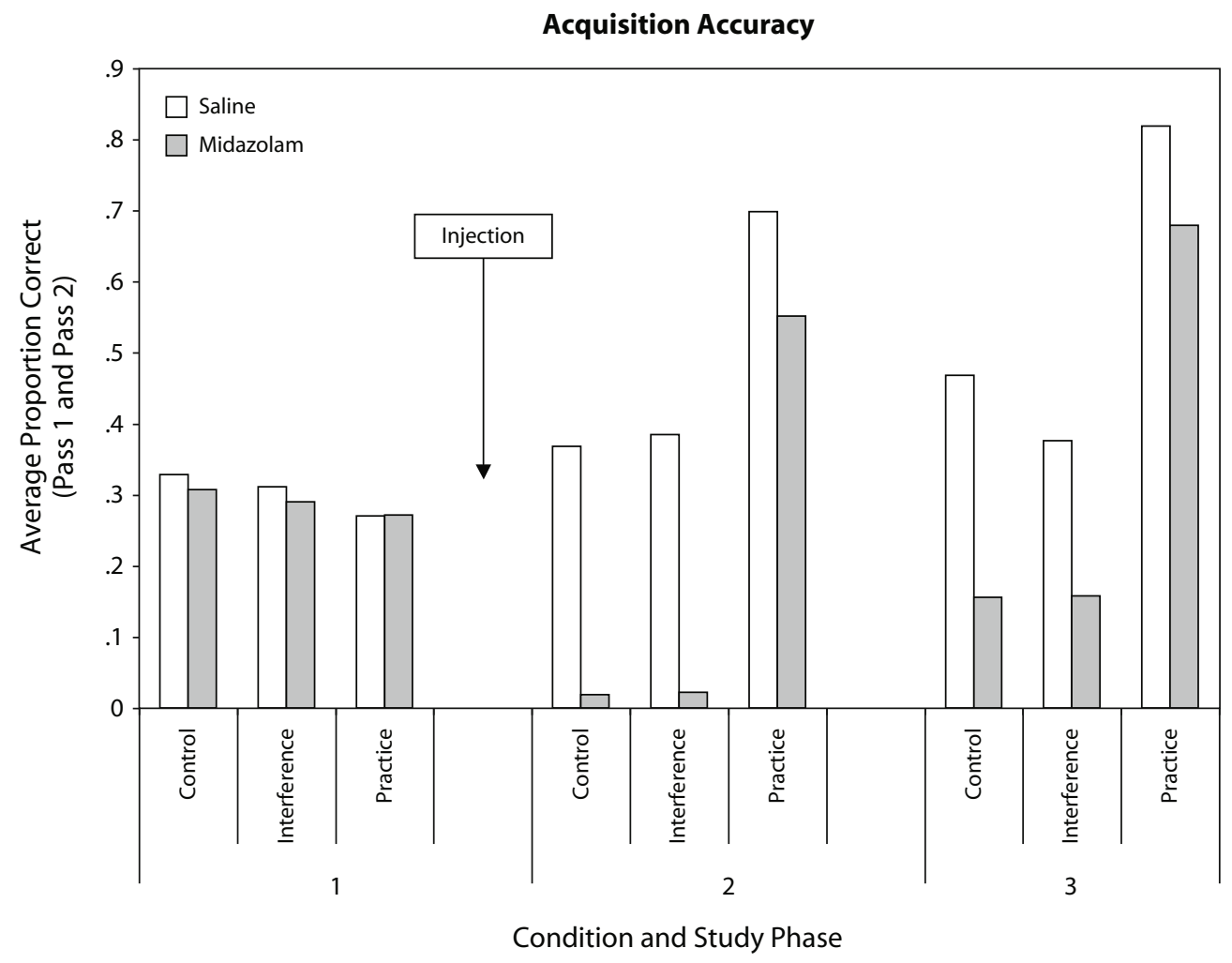

Figure 1. Proportion correct during acquisition of the word pairs for each list as a function of type of pair and drug condition, averaged over the two passes per list.

and interference condition, but not the practice pairs that were relatively unaffected by the drug to begin with.

It is also noteworthy that there is an interference effect in the saline condition for List $3[F(1,20)=18.0, p<$ $.001]$, such that subjects were less accurate in learning the interference pairs than the control pairs. That effect is not present for acquisition of List 2, presumably because interference was greater with the addition of List 3. This pattern is not observed in the midazolam condition.

In sum, midazolam had the desired effect of blocking acquisition of new information. In the saline condition, practice pairs were learned best and interference pairs were learned worst. We can now ask how final recall (retention) of the material learned prior to injection was affected by the differential ability to learn pairs on the postinjection lists.

\section{Final Recall}

Figure 2 presents the data from the final test phase, after subjects had studied all three lists. There was a main effect of drug condition $[F(1,30)=67.9, p<.001]$, such that recall was better in the saline condition, and a main effect of stimulus type $[F(6,180)=88.3, p<.001]$, such that practice pairs were better recalled than the other two. There was a list $\times$ drug interaction $[F(2,60)=70.3, p<$ $.001]$, such that recall was much better for List 1 than for the other lists in the midazolam condition, but not in the saline condition.

Of particular interest is how "retrograde facilitation" varied for the 3 types of pairs. Consistent with previous re- search, more List 1 pairs were correctly recalled in the midazolam condition than for the saline condition $[F(1,30)=$ $4.1, p<.05]$, demonstrating retrograde facilitation. This effect was not reliable for the control condition $[F(1,30)=$ $2.2, p>.05]$, although the direction of the effect was the same as in previous research. The specific interference pairs, on the other hand, were significantly better recalled in the midazolam condition than in the saline condition for List $1[F(1,30)=16.5, p<.001]$, and the interaction between control and specific interference by drug condition was reliable $[F(1,30)=4.1, p<.05]$. This interaction was driven by the result that, for saline, there was a reliable recall advantage of control pairs compared with the interference pairs $[F(5,150)=23.7, p<.001]$.

The latency data (time to initiate the cued recall for correct responses), shown in Figure 3 in an analogous fashion to Figure 2, are largely consistent with the accuracy data. There was no main effect of drug condition on response time (RT) $(F<1)$, but subjects were significantly faster for practice pairs than for other pair types $[F(1,30)=$ $116.6, p<.001] .^{2}$ The critical comparisons are between the saline and midazolam conditions for the control and interference pairs on List 1. For the control pairs, there is no significant difference in RT between saline and midazolam, with the latter slightly slower than the former. On the other hand, RTs are significantly faster in the midazolam condition than in the saline condition for the specific interference pairs $[F(1,30)=16.5, p<.001]$, and, as in the case of the accuracy data, the interaction between 


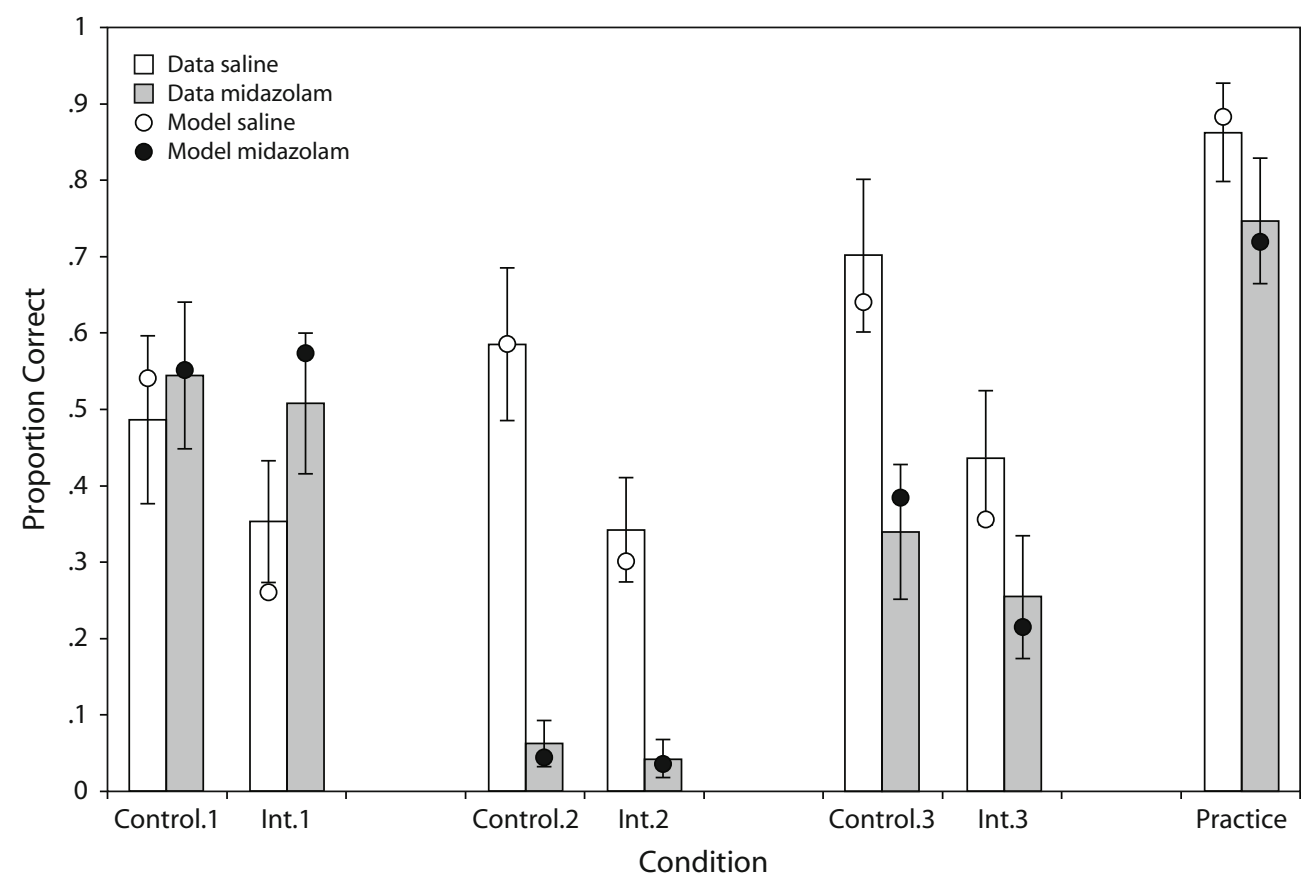

Figure 2. Proportion correct on the final test for each pair type for each list, as a function of drug condition (practice pairs have the same response regardless of list and so are collapsed over list). The model estimated data points are superimposed.

drug condition and pair type was significant $[F(1,30)=$ $9.0, p<.05]$. These results reinforce the view that the retrograde facilitation for items learned prior to an injection of midazolam is greatest for those items that would otherwise suffer specific interference.

\section{DISCUSSION}

Because it is difficult to manipulate general interference, laboratory studies have tended to focus on specific interference, or cue overload. We were able to manipulate general interference without introducing confounds resulting from amount of sleep, time of day, or delay. That aspect of our study was a replication of other research that examined the effects of general interference by also using a drug that produces transient anterograde amnesia. What set our study apart is the fact that we compared the retrograde facilitation produced by the absence of general interference with the facilitation produced by the absence of both specific and general interference; that is, our specific interference condition also contained the same general contextual interference of the control condition. We found significantly more retrograde facilitation for the condition that included both specific interference and general interference than for the control condition, which suffered from general contextual interference only. Facilitation was assessed by comparing retention after studying under midazolam versus under saline. When pairs were studied under saline, performance was worse in the specific interference condition than in the control condition, whereas performance did not vary for those two conditions under midazolam.
As a test of whether these results can be understood without assuming that new learning blocks consolidation, we attempted to fit these data with a memory model that did not include a consolidation process. ${ }^{3}$ We used a set of processing assumptions and a representation that had been used to fit a number of other memory experiments, importing the parameter estimates from previous models. Below, we briefly explain the assumptions of the model and how we fit these data.

\section{Fitting the Data With a Computational Model}

Figures 2 and 3 also plot the theoretical data points derived from the Source of Activation Confusion (SAC) model for the dependent measures of accuracy and latency (illustrated by superimposed points on the bar charts). The quality of these fits is noteworthy, because they were accomplished with few parameter estimates beyond those used previously to fit other memory experiments. The assumptions and equations of the model have been described elsewhere (e.g., Reder et al., 2000), so only a brief description will be given here, focusing on novel assumptions required to model this experiment. The more basic model assumptions, a pointer to the spreadsheet of the model available online, and the model fit for the list confusion errors can all be found in the Appendix.

We assume that information is represented in the mind as a network of interassociated concepts (nodes). These concepts and their associations vary in strength as a function of prior history of exposure, gaining strength with repetitions, losing strength between repetitions. It is the detailed specification of how representations change with experience and how activation values are interpreted in 


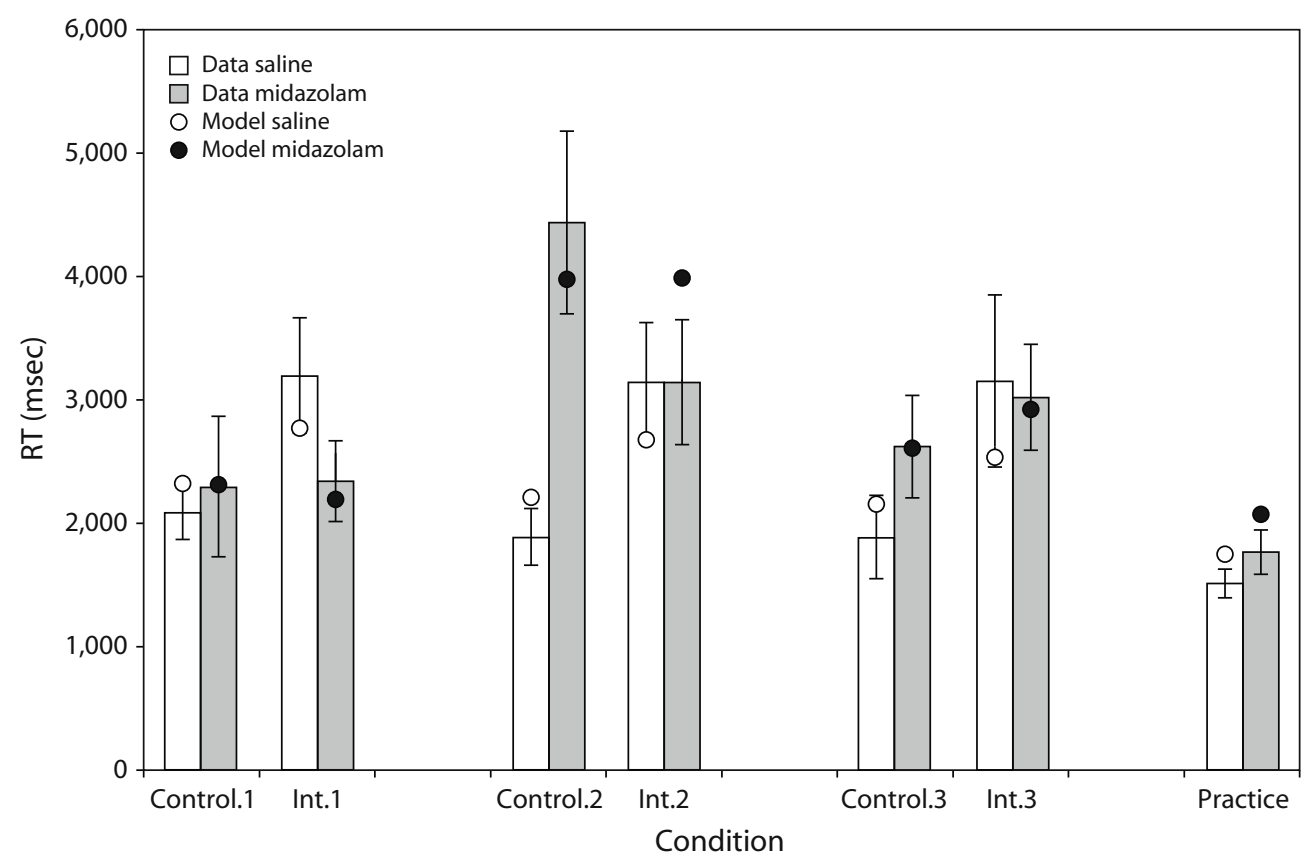

Figure 3. Mean time to correctly respond on the final test as a function of type of pair, list, and drug condition. The model estimated data points are superimposed.

particular situations that allows SAC to make specific, quantifiable predictions for many types of tasks. ${ }^{4}$

Figure 4 provides a schematic illustration of the memory representation for the saline and midazolam conditions for two interference word pairs on List 1 (before there is specific interference) and List 2. Ovals represent concepts such as words, the experimental context, and the episode that associates a stimulus with its response words for a particular list in this experiment. The memory strength of the pair is represented by the episode node's level of activation and the strength of the binding from the words in the pair to the node that binds them. Each time a pair is repeated, the node and its links are strengthened, decaying in strength with the passage of time since the presentation.

We simulate the subject's experience at test of being given the cue word and list number by activating the corresponding word node and list node and also by activating the general experimental context node that we assume that subjects tacitly activate in the experiment. Activation spreads from these three sources to all associated nodes in proportion to their relative strength. The response term will be recalled if the activation level of the correct episode node passes threshold.

In the midazolam condition, we assume that temporary amnesia is caused by the inability to create new bindings (Ghoneim, 2004; Park, Quinlan, Thornton, \& Reder, 2004; Reder et al., 2006). We assume that after the injection of midazolam, subjects have a decreased ability to form links. The effect of the drug is assumed to decay exponentially, meaning that the probability of forming a new link is

$$
P(\text { encoding })=1-C_{m} \cdot 2^{-\frac{t_{\text {injection }}}{t_{h l}}},
$$

where $t_{h l}$ is the halflife of the drug (Albrecht et al., 1999). The dashed lines in Figure 4 represent those links that are rarely formed under midazolam. As a consequence, there will be fewer links from the experimental context node (a reduction in general interference), as well as fewer links from the cue words of the interference pairs learned on List 1 in the midazolam condition than in the saline condition. That means that there should be less specific interference for interference pairs in the midazolam condition, since the potentially competing associations were rarely formed.

With these assumptions, we were able to fit not only the accuracy data (the dots superimposed on Figure 2) but also the RT data (dots on Figure 3) and the list-confusion errors and accuracy (Figures A1 and A2 in the Appendix). RTs were estimated with only two additional parameters by using the activation value of the relevant episode node used to fit accuracy data.

In summary, our model provided an excellent fit to these data without assuming any role for consolidation. Our explanation for retrograde facilitation in the midazolam condition is based on fewer bindings being formed that would otherwise share the activation that spreads from the cue words (sources of activation). The greater facilitation under midazolam for pairs in the specific interference condition than for pairs in the control condition is explained by having less competition from two sources - the stimulus term and the general experimental context; the control items only have less competition from the general context.

\section{General Contextual Interference and Cue Overload Revisited}

Wixted's (2004) recent Annual Review article has drawn attention to the importance of general interference as a cause 

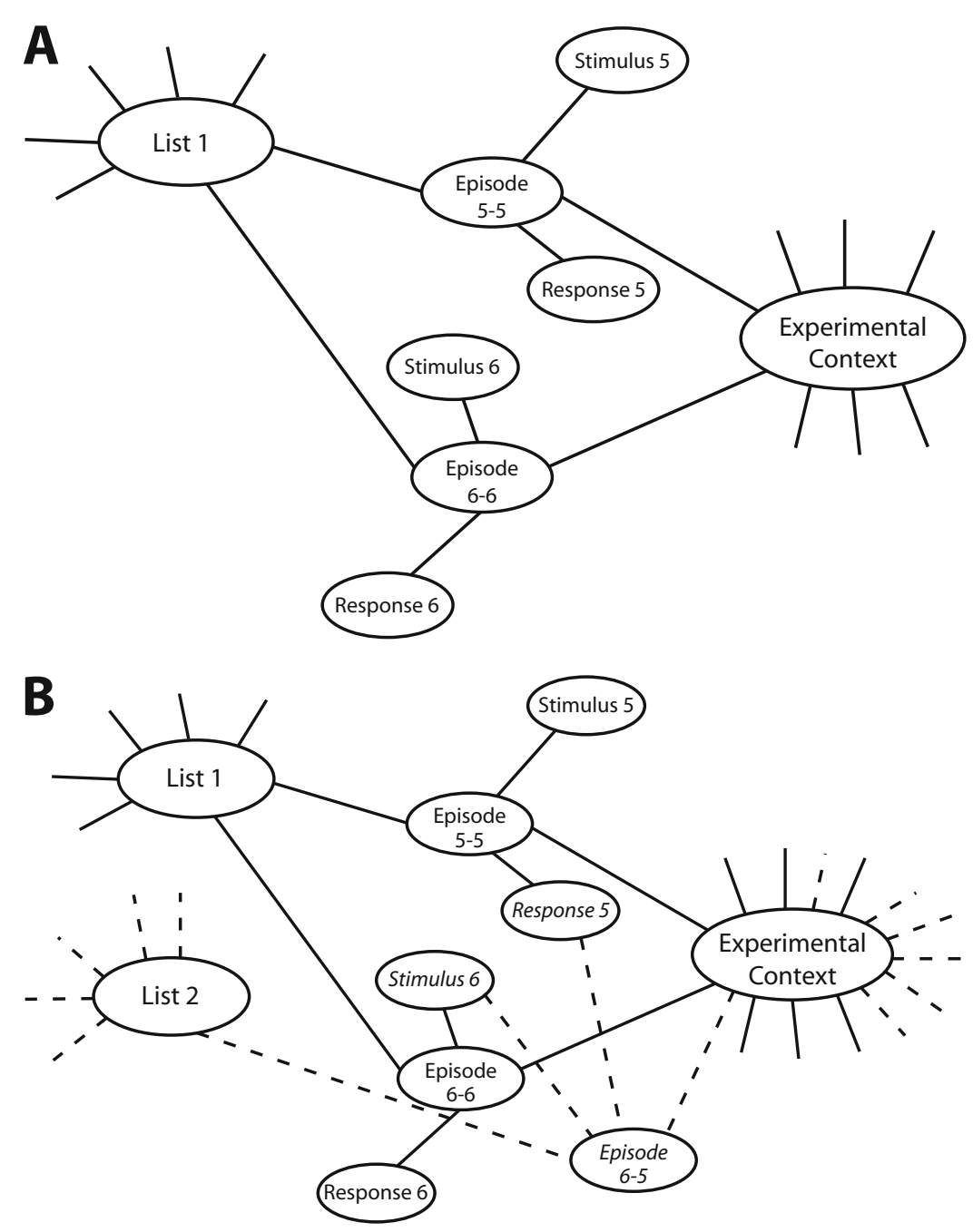

Figure 4. Illustration of model representation of interference pairs learned during (A) List 1 (preinjection) and (B) List 2 (postinjection). The associations for this list are dashed to denote that links are not likely to be formed in the midazolam condition.

of forgetting and highlighted the degree to which memory researchers have ignored its contributions. He has cogently argued that the role of cue overload as a mechanism responsible for forgetting is overrated. As he points out, much of what is learned is forgotten, even when specific interference plays no role in forgetting. Nonetheless, it is important to note that Wixted (2004) strongly agrees that performance under saline will be worse in a condition with two sources of interference than in a condition with only one. Therefore, our findings are not inconsistent with his position.

The difference between the drug conditions for general interference was smaller in our study compared with the ones that Wixted (2004) described. This probably occurred because our experiment used cued recall rather than free recall, a pattern our model would predict. SAC posits that with cued recall there is an additional source of activation to make the episode node accessible, thereby minimizing the role of the general experimental context. Our simulation showed only a very small advantage for midazolam in the general context condition, but SAC would predict a larger difference between drug conditions for general interference, if there were no cue words to provide an additional strong source of activation; that is, the difference in the amount of competition from the general context node would play a larger role in a free-recall paradigm.

General interference is an important source of forgetting, but it is clear that specific interference is another, and that two sources of interference are more disruptive than one. Although an explanation for retrograde facilitation with benzodiazepines that is based on less disruption of the consolidation process is plausible, the results of our experiment indicate that such an account is unnecessary. ${ }^{5}$

\section{AUTHOR NOTE}

This work was supported by Grants 2-R01-MH52808 and R01MH068243 from the National Institute of Mental Health. We thank John Wixted for comments on an earlier version of the manuscript, and the Nurse Anesthesia Program, University of Pittsburgh School of Nursing, for providing nurses. Correspondence concerning this article should be addressed to L. M. Reder, Psychology Department, Carnegie Mellon University, 5000 Forbes Avenue, Pittsburgh, PA 15213 (e-mail: reder@cmu.edu). 


\section{REFERENCES}

Albrecht, S., Ihmsen, H., Hering, W., Geisslinger, G., Dingemanse, J., SCHWILdEN, H., \& SchütTLER, J. (1999). The effect of age on the pharmacokinetics and pharmacodynamics of midazolam. Clinical Pharmacology \& Therapeutics, 65, 630-639.

ANDERSON, J. R. (1974). Retrieval of propositional information from long-term memory. Cognitive Psychology, 5, 451-474.

GhoneIm, M. M. (2004). Drugs and human memory: Clinical, theoretical, and methodologic issues. Anesthesiology, 100, 987-1002; 1277-1297.

Park, H., Quinlan, J. J., Thornton, E. R., \& Reder, L. M. (2004). The effect of midazolam on visual search: Implications for understanding amnesia. Proceedings of the National Academy of Sciences, 101, 17879-17883.

Reder, L. M., Nhouyvanisvong, A., Schunn, C. D., Ayers, M. S., Angstadt, P., \& Hiraki, K. (2000). A mechanistic account of the mirror effect for word frequency: A computational model of remember-know judgments in a continuous recognition paradigm. Journal of Experimental Psychology: Learning, Memory, \& Cognition, 26, 294-320.

Reder, L. M., OAtes, J. M., Thornton, E. R., Quinlan, J. J., Kaufer, A., \& SAUER, J. (2006). Drug-induced amnesia hurts recognition, but only for memories that can be unitized. Psychological Science, 17, 562-567.

REDER, L. M., \& Ross, B. H. (1983). Integrated knowledge in different tasks: The role of retrieval strategy on fan effects. Journal of Experimental Psychology: Learning, Memory, \& Cognition, 9, 55-72.

WiXTED, J. T. (2004). The psychology and neuroscience of forgetting. Annual Review of Psychology, 55, 235-269.

Wixted, J. T. (2005). A theory about why we forget what we once knew. Current Directions in Psychological Science, 14, 6-9.

\section{NOTES}

1. Performance improved from the first cycle to the second cycle for all three lists and was highly reliable $[F(1,30)=99.8, p<.001]$, but is not discussed to save space.
2. As can be seen in Figure 2, there were few correct RTs for pairs learned on List 2 in the midazolam condition (practice pairs were already learned), and as a result there were few correct RTs in those conditions, forcing us to estimate RTs for missing cells. We do not consider the RT data in the midazolam condition, especially for List 2, to be very accurate. However, the RT data for List 1, learned prior to drug administration, have few errors and do not suffer from this problem.

3. We are not claiming that consolidation is not an important process in memory formation. Rather, we are disputing whether the facilitation effects reported in this study are caused by differential consolidation.

4. SAC was originally adapted from an earlier version of ACT-R (Anderson, 1974); therefore, most of the equations are very similar to those used in ACT-R. However, a number of important assumptions are different from ACT-R

5. Wixted (2004) notes that there is a temporal gradient in the effectiveness of sleep (or any similar intervention) that blocks formation of new memories, so that facilitation is greatest if sleep occurs immediately after learning. In our model, this temporal gradient would be produced if the words presented right after the injection were associated with the same context node as words learned immediately prior to the injection, whereas words learned later would be more likely to be associated with a different context node

\section{ARCHIVED MATERIALS}

The following materials [and links] associated with this article may be accessed through the Psychonomic Society's Norms, Stimuli, and Data archive, www.psychonomic.org/archive.

To access these files [or links], search the archive for this article using the journal name (Psychonomic Bulletin \& Review), the first author's name (Reder), and the publication year (2007).

FILE: Reder-PB\&R-2007.zip

DESCRIPTION: The compressed archive file contains one file, PA Recall_halflife_final.sls, an Excel file that implements the SAC Model of Midazolam Paired Associates (Recall).

AUTHOR's E-MAIL ADDRESS: reder@cmu.edu.

AUTHOR's WeB SITE: www.andres.cmu.edu/user/reder. 


\section{APPENDIX}

The SAC equations used to model the cued-recall data (accuracy, latency, and type of errors) are listed in Table A1. We implemented this model using Excel and the model that fits these data can be found online at www.andrew.cmu .edu/user/reder/model_fits/PAmidazolam.html, and also at psychonomic.org/archive. A few of the equations are complex because the model is a process model that is usually implemented in Lisp rather than Excel. Knowing that Lisp would not be a prerequisite to examining the model, we chose to model this time in Excel (most equations are easily described) so that our model would be more accessible.

Table A1

SAC Model Parameter Descriptions, Fixed Constants and Model Equations

\begin{tabular}{clc}
\hline Parameter Name & \multicolumn{1}{c}{ Function } & Value \\
\hline$A_{\text {boost }}$ & Current activation from perceptual boost & 40 \\
$d_{n}$ & Power-law decay constant for base level activation & 0.175 \\
$d_{l}$ & Power-law decay constant for link strength & 0.12 \\
$c_{n}$ & Strength constant of a node & 25 \\
delay & Average time delay between study and test & 100 min \\
$B_{0}$ & Base level activation (Kučera \& Francis, 1967 word frequency average of 90) & $90^{0.4}$ \\
$F_{0}$ & Preexisting fan effect (Kučera \& Francis, 1967 word frequency average of 90) & $90^{0.7}$ \\
$\sigma_{\text {episode }}$ & Episode (recollection) activation standard deviation & $0.357^{*}$ \\
$\tau_{\text {episode }}$ & Episode (recollection) activation threshold & $4.517^{*}$ \\
$C_{m}$ & Effectiveness of midazolam immediately after injection & $1^{*}$ \\
$T_{h l}$ & Rate the drug gets to half potency (memorial effects) & $31 \mathrm{~min}^{*}$ \\
$P_{\text {sr }}$ & Probability of spurious recollection & $0.499^{*}$ \\
$A_{\text {max }}$ & Activation cap & $89.3^{*}$ \\
\hline
\end{tabular}

*The five parameters with asterisks were fitted to these data. The others were imported from previous models.

Activation spreads between nodes via links. The current activation level of a node can rise from environmental stimulation or activation received from associated nodes. The increase in activation of a receiving node $R$, which has received activation from other nodes, is computed by summing the activation it is receiving from all source nodes; however, how much each source node sends depends on (1) that source node's strength, and (2) how much competition the connection to $R$ has from other links associated with that source. More competing links and stronger competing links, relative to the strength of the critical link, lead to less activation reaching the receiving node. This property gives the model the ability to simulate fan effects (e.g., Anderson, 1974; Reder \& Ross, 1983).

As a simplifying assumption, we assume that a response term will be recalled if the node that binds them passes threshold. If more than one episode node is above threshold for any given item (e.g., in the interference condition in which there are links that go to multiple episode nodes), we assume that the response is selected randomly from the possible response nodes with equal probability. Thus, there is some tendency to recall the wrong response for an interference pair; however, the correct episode node is more likely to get over threshold because it has an additional source of activation, namely the list cue $(1,2$, or 3$)$. On the other hand, there is also some tendency to inadvertently produce additional hits for the practice pairs (retrieving the association from the wrong list but still gives the correct response). Those two equations are the only ones that are complicated and should be examined on the Excel spreadsheet on the Web site.

We also assume that a node is not strengthened when its current activation is above a specific level. This assumption is viewed as a proxy for habituation, so that when the same information is experienced over and over it no longer attracts as much attention and does not gain strength indefinitely.

Table A2

SAC Model Parameter Descriptions, Fixed Constants, and Model Equations

\begin{tabular}{|c|c|}
\hline Equation & Description \\
\hline $\begin{array}{l}\text { (1) } B=B_{0}+c_{n} \cdot \text { delay }^{-d_{n}} \\
\text { (2) } S=\text { delay }^{-d_{l}} \\
\text { (3) } A_{\text {cue }}=B+A_{\text {boost }}\end{array}$ & $\begin{array}{l}\text { Base level activation as a function of normative strength and delay } \\
\text { Link strength decaying as a function of delay } \\
\text { Cue node activation as a function of base level and current boost }\end{array}$ \\
\hline (4) $A_{\text {input }}=\sum_{\text {cue }}\left(A_{\text {cue }} \cdot \frac{S_{\text {cue,episode }}}{\sum S_{\text {cue }}}\right)$ & $\begin{array}{l}\text { Boost in episode node's current strength due to spreading activation from } \\
\text { cues at test }\end{array}$ \\
\hline (5) $A_{\text {episode }}=\ln \left(B+A_{\text {input }}\right)$ & $\begin{array}{l}\text { Current activation is the natural logarithm of the sum of base level activa- } \\
\text { tion and received spreading activation }\end{array}$ \\
\hline (6) $P($ episode $)=N\left(A_{\text {episode }} \mid \delta_{\text {episode }}, \tau_{\text {episode }}\right)$ & $\begin{array}{l}\text { Probability of the episode node being above threshold as a function of } \\
\text { the cumulative normal distribution at its activation }\end{array}$ \\
\hline (7) $P($ encoding $)=1-C_{m} \cdot 2^{-\frac{t_{\text {injection }}}{t_{h l}}}$ & Probability of forming a new link under midazolam \\
\hline
\end{tabular}

Five parameters were varied to fit the pattern of responses (correct or error) which contained 42 data points, for an RMSD of 0.046 and an $R^{2}$ of .94 (Figures A1 and A2). Those parameter values were evaluated against the test accuracy data containing 14 data points for an RMSD of 0.061 and an $R^{2}$ of .94 (Figure 3). The RT data were fit with two additional parameters that translate node activation values to RT using the equation for converting activation to RT: RT $=$ $C * \exp (-D * \ln \{A\})$, where $C$ and $D$ are fitted parameters and $A$ is the activation value derived from fitting the accuracy data. The fit was quite good with only these two additional parameters for an RMSD of 433.8 (in msec) and an $R^{2}$ of .83 . 
APPENDIX (Continued)

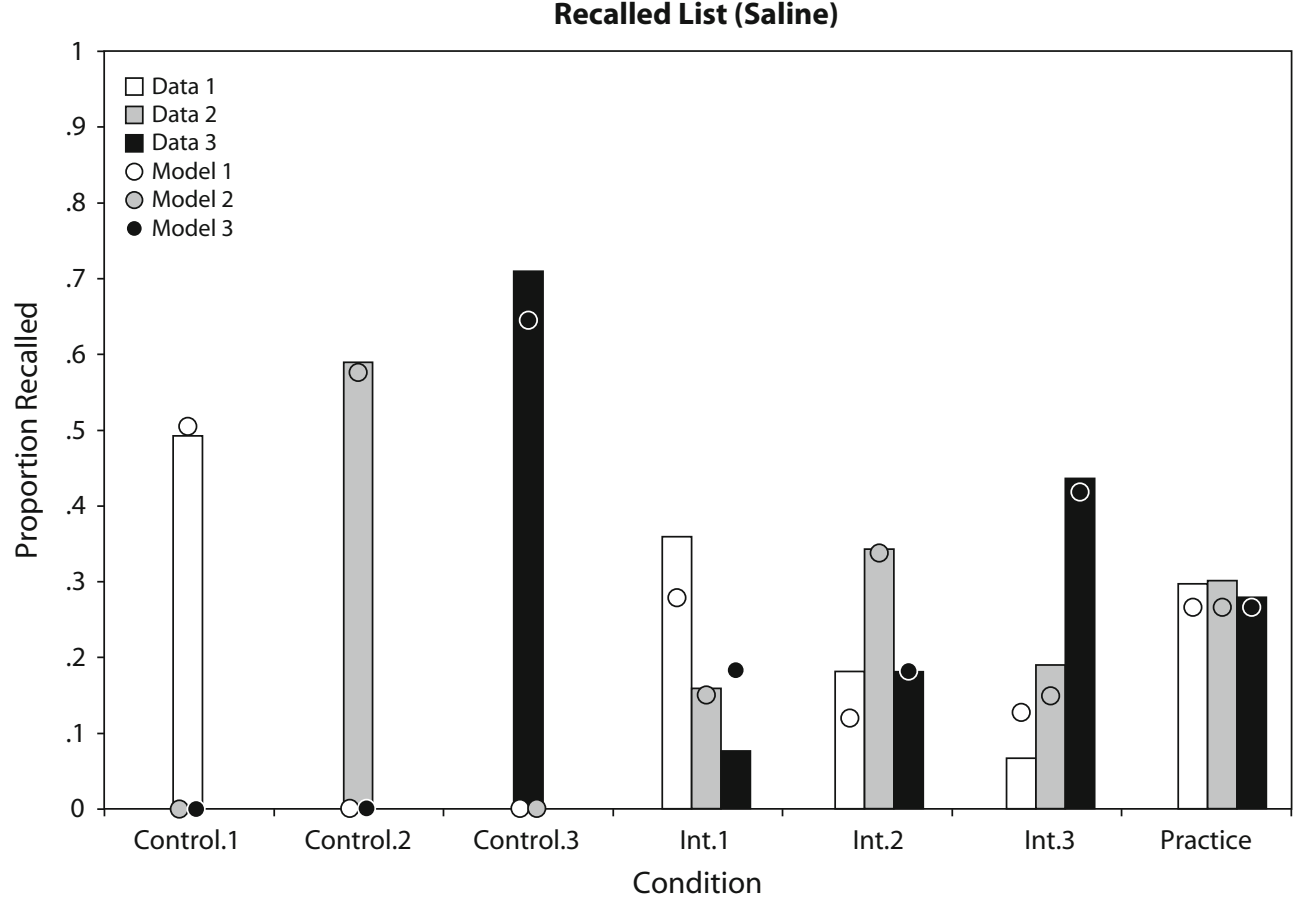

Figure A1. Proportion of responses in the saline condition for correct list and wrong lists as a function of type of pair and list. The model estimated data points are superimposed.

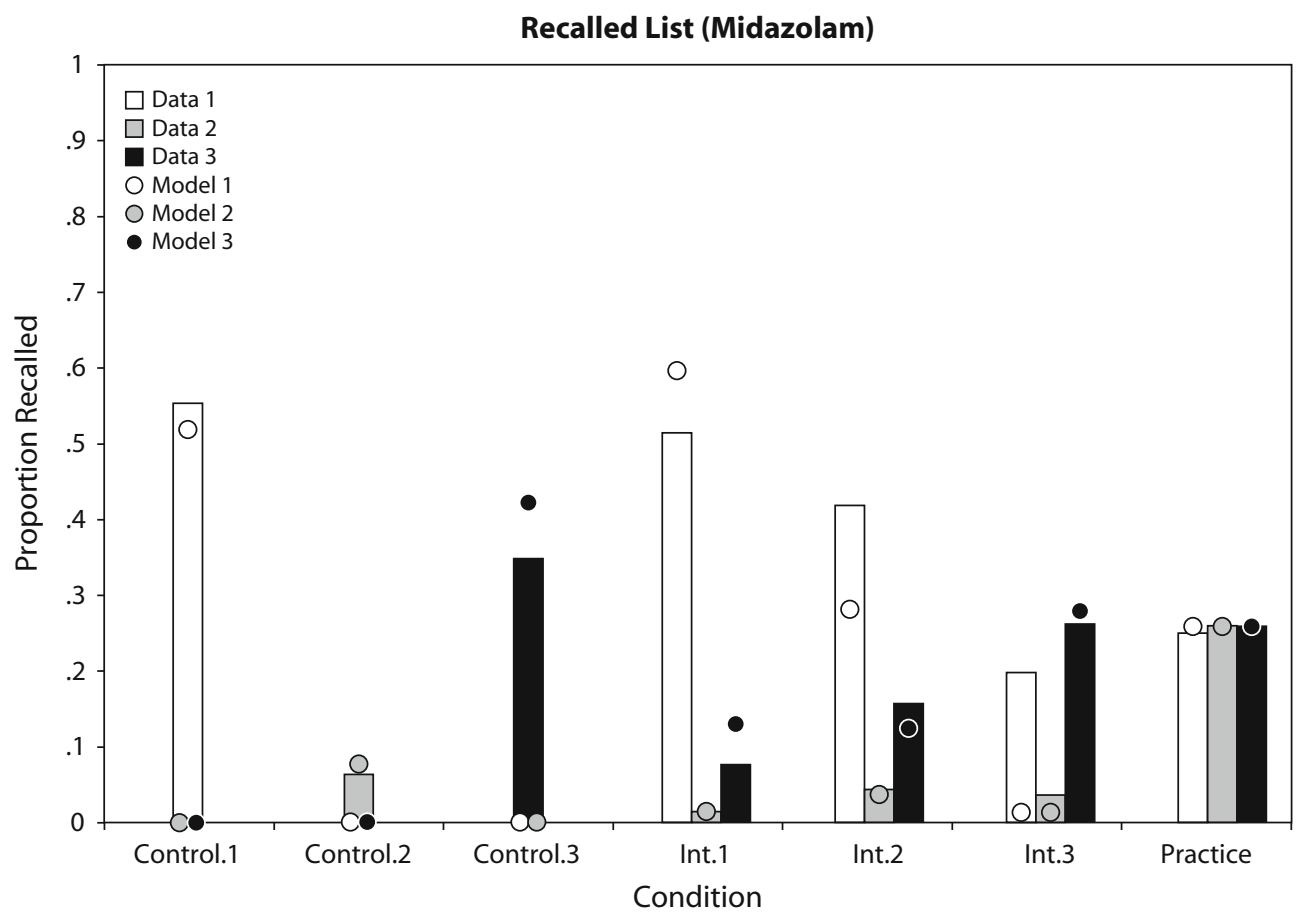

Figure A2. Proportion of responses in the midazolam condition for correct list and wrong lists as a function of type of pair and list. The model estimated data points are superimposed.

(Manuscript received January 18, 2006; revision accepted for publication May 11, 2006.) 